\title{
CHOQUET AGGREGATION BASED DECISION MAKING UNDER Z-INFORMATION
}

\author{
Lala M. Zeinalova \\ Department of Computer-Aided Control Systems, Azerbaijan State Oil Academy, Azerbaijan \\ E-mail: Zeynalova-69@mail.ru
}

\begin{abstract}
The concept of decision making under uncertainty is usually associated with information that may be incomplete, not reliable or imprecise, so there are several types of uncertainty. A partial absence of beliefs and fuzziness are some of the aspects of uncertainty. In this paper we consider a somewhat different framework for representing our knowledge. Zadeh suggested a Z-number notion, based on a reliability of the given information. In this study we apply Zinformation to decision making in business problem and suggest the framework for decision making on a base of Z-numbers. The method associates with the construction of a non-additive measure as a lower prevision and uses this capacity in Choquet integral for constructing a utility function. An example of real-world business problem is used to illustrate the proposed approach.
\end{abstract}

Keywords:

Z-Number, Fuzzy Set, Choquet Integral, Lower Prevision, Decision Making

\section{INTRODUCTION}

Depending upon structure of available information, there are a large number of decision-making methods. One of the well-known methods is the one of the expected utility proposed by von Neumann and Morgenstern [1], and subjective expected utility by Savage [2]. Expected Utility Theory states that the decision maker chooses between risky or uncertain prospects by comparing their expected utility values, i.e., the weighted sums obtained by adding the utility values of outcomes multiplied by their respective probabilities [3]. There are two well-received versions of the theory, i.e., Subjective Expected Utility Theory in the case of uncertainty, and von Neumann- Morgenstern Theory in the case of risk [3]. These approaches require that the objective or subjective probabilities and utility values be exactly known. But in real-world in many cases it becomes impossible to determine the values of objective probabilities [4]. It is more plausible to determine the values of subjective probabilities, reflecting the beliefs of a decision maker. Imprecision and uncertainty may be in the aspects of measurement, probability, or descriptions [5]. Imprecision in description is the type of imprecision addressed by fuzzy logic [5]. It is the ambiguity, vagueness, qualitativeness, or subjectivity in natural language [5]. In all existing classical decision making theories the probability measures are regarded to be described in a precise manner, which, in many real-world cases could be impossible to achieve. In real-world decision making problems, especially those encountered in economics, such probabilities are subjective and usually imprecise. There are a lot of approaches for describing imprecision of probability relevant information. One of the approaches is the use of hierarchical imprecise models. These models capture the second-order uncertainty inherent in real problems. According this approach an expert opinion on probability assessments is usually imprecise $[6,7,8]$. The method, proposed in [7] uses a Choquet integral for determination the values of utility functions for further comparing the preferences among acts. The authors construct a low prevision as non-additive measure a uses this capacity in Choquet integral. The Choquet integral has many advantages: it is continuous, non decreasing, located between min and max. The major advantage of the Choquet integral is the use of fuzzy measure [9] for an estimation of relation between the different states of nature. In [7] an imprecise hierarchical decision-making model has the first and the second levels described by interval probabilities. In [8], where a hierarchical uncertainty model which exhibits imprecision at its second level in sense of the use of lower probabilities at this level is represented. First level of this model may be either precise or imprecise. Author shows that no matter whether the first level is precise or imprecise the suggested hierarchical model has the same implications for decision analysis and decision reasoning. The model is a generalization of imprecise probabilities, Bayesian models and fuzzy probabilities. However one should mention that this model doesn`t deal with probability distribution (multiple priors) which are more general description of incomplete probability relevant information.

In [10] Zadeh introduced a concept of Z-numbers to describe information which is uncertain, incomplete or partially truth. A Znumber is a pair of fuzzy numbers $(\tilde{A}, \tilde{R})$. Here $\tilde{A}$ is a fuzzy value of some variable and $\tilde{R}$ is a fuzzy reliability or a fuzzy probability for this value [11]. Zadeh suggests some operations for computation with Z-numbers, using the extension principle. In [12] author shows how to use Z-number based information for decision making. In this case Z-information is given in terms in of a Dempster-Shafer belief structure and in terms of type- 2 fuzzy sets. In [13] authors considered multi-criteria decision making problem under Z-information. They don't use any operations over the Z-numbers using extension principle but suggest easier method, converting the Z-numbers to classical fuzzy numbers and determining a weight for each alternative.

In this study we suggest generalization of Choquet integral for environment described by Z-valuation of the uncertain information. We provide as example a real-world business problem used to illustrate the proposed approach.

The paper is organized as follows. In section 2 we present required preliminaries and cover some prerequisite material. In section 3 we formulate a statement of the problem. In section 4 we present a method used to solve it. In section 5 we cover application of the suggested method to a real-life business problem. Concluding comments are included in section 6 .

\section{PRELIMINARIES}

\section{Definition 1: Fuzzy sets [14].}

Let $X$ be a classical set of objects, called the universe, whose generic elements are denoted $x$. Membership in a classical subset 
$A$ of $X$ is often viewed as a characteristic function $\mu_{A}$ from $X$ to $\{0,1\}$ such that,

$$
\mu_{A}(x)= \begin{cases}1 & \text { iff } x \in A \\ 0 & \text { iff } x \notin A\end{cases}
$$

where, $\{0,1\}$ is called a valuation set; 1 indicates membership while 0 - non membership.

If the valuation set is allowed to be in the real interval $[0,1]$, then $A$ is called a fuzzy set, $\mu_{\mathrm{A}}$ is the grade of membership of $x$ in $A: \mu_{A}(x): X \rightarrow[0,1]$.

\section{Definition 2: A Z-number [10].}

A Z-number is an ordered pair of fuzzy numbers, $(\tilde{A}, \tilde{R}) . \tilde{A}$ is a fuzzy restriction on the values which a real-valued uncertain variable is allowed to take. $\tilde{R}$ is a measure of reliability of the first component.

\section{Definition 3: Choquet integral[15-20].}

Let $\varphi: \Omega \rightarrow R$ be a measurable real-valued function on $\Omega$ and $\eta: F \rightarrow[0,1]$ be a non-additive measure defined over $F$. The Choquet integral of $\varphi$ with respect to $\eta$ is defined as

$$
\int_{\Omega} \varphi d \eta=\sum_{i=1}^{n}\left(\eta\left(B_{(i)}\right)-\eta\left(B_{(i-1)}\right) \varphi\left(\omega_{(i)}\right)\right)
$$

where, index $(i)$ implies that elements $\omega_{i} \in \Omega, i=1, \ldots, n$ are permuted such that $\varphi\left(\omega_{(i)}\right) \geq \varphi\left(\omega_{(i+1)}\right), \varphi\left(\omega_{(n+1)}\right)=0$ and $B_{(i)}=\left\{\omega_{(1)}, \ldots, \omega_{i)}\right\} \subseteq \Omega$.

A value of fuzzy utility function for an action is determined as a fuzzy number-valued Choquet integral

$$
\int_{\Omega} \tilde{\varphi} d \tilde{\eta}=\sum_{i=1}^{n}\left(\tilde{\eta}\left(B_{(i)}\right)-\tilde{\eta}\left(B_{(i-1)}\right) \tilde{\varphi}\left(\omega_{(i)}\right)\right.
$$

where, (i) means that utilities are ranked such that $\tilde{\varphi}\left(\omega_{(1)}\right) \geq \ldots \geq \tilde{\varphi}\left(\omega_{(n)}\right), \tilde{\varphi}\left(\omega_{(n+1)}\right)=0$.

Definition 4: Fuzzy measure [15, 21].

Let $\varepsilon^{n}$ be a space of all fuzzy subsets of $R^{n}$. These subsets satisfy the conditions of normality, convexity, and are upper semicontinuous with compact support. Let $\tilde{V}, \tilde{W} \in \varepsilon^{n}$. A fuzzy number-valued fuzzy measure $((z)$ fuzzy measure $)$ on $\tilde{F}$ is a fuzzy number-valued fuzzy set function $\tilde{\eta}: \tilde{F}$ with the properties:

1) $\eta(\phi)=0$

2) if $\tilde{V} \subset \tilde{W}$ then $\tilde{\eta}(\tilde{V}) \leq \tilde{\eta}(\tilde{W})$;

3) if $\tilde{V}_{1} \subset \tilde{V}_{2} \subset . ., \tilde{V}_{n} \subset . . \in \tilde{F}$, then $\tilde{\eta}\left(\cup_{n-1}^{\infty} \tilde{V}_{n}\right)=\lim _{n \rightarrow \infty} \tilde{\eta}\left(\tilde{V}_{n}\right)$;

4) if $\tilde{V}_{1} \supset \tilde{V}_{2} \supset \ldots, \tilde{V}_{n} \in \tilde{F}$, and there exists $n_{0}$ such that $\tilde{\eta}\left(\tilde{V}_{n_{0}}\right) \neq \tilde{\infty}$, then $\tilde{\eta}\left(\bigcap_{n-1}^{\infty} \tilde{V}_{n}\right)=\lim _{n \rightarrow \infty} \tilde{\eta}\left(\tilde{V}_{n}\right)$.

Definition 5: Lower prevision [22 - 27].

A coherent lower prevision is defined as a lower expectation functional from the set of gambles to the real numbers that satisfies some rationality criteria. This function is conjugate to another that is called a coherent upper prevision. When a coherent lower prevision coincides with its conjugate coherent upper prevision we call it a linear prevision. An unconditional lower prevision $\underline{P}(X)$ is coherent if and only if it is the lower envelope of dominating linear previsions.

If the lower prevision $\underline{P}$ is represented as the lower envelope of a closed convex set $P$ of linear previsions then

$$
\underline{P}=\min \{P(X): X \subset S\}
$$

Lower prevision $\underline{P}$ is characterized by probability density function of each linear prevision in extreme points [28].

In particular case, when linear prevision is a probability measure the lower prevision is the lower envelope of multiple priors. In this work we use lower prevision as non-additive measure. So we can define $\underline{P}$ as $\eta$.

\section{PROBLEM STATEMENT}

A set of acts $f_{1}, f_{2}, \ldots, f_{n}$ with a number of possible utilities

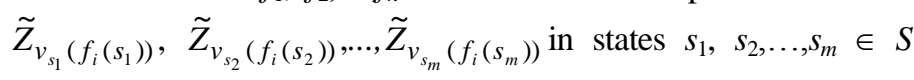
and the corresponding state probabilities $\tilde{Z}_{P\left(s_{1}\right)}, \tilde{Z}_{P\left(s_{2}\right)}, \ldots, \tilde{Z}_{P\left(s_{m}\right)}$ are given and described by Z-numbers (Table.1 and Table.2). Then we can determine the value of utility function for each act.

Table.1. The payoff table with utilities as Z-numbers

\begin{tabular}{|c|c|c|c|c|}
\hline & $s_{1}$ & $s_{2}$ & $\ldots$ & $s_{m}$ \\
\hline$f_{1}$ & $\left(\tilde{v}_{s_{1}}\left(f_{1}\left(s_{1}\right)\right), \tilde{R}_{1}\right)$ & $\left(\tilde{v}_{s_{2}}\left(f_{1}\left(s_{2}\right)\right), \tilde{R}_{1}\right)$ & -- & $\left(\tilde{v}_{s_{m}}\left(f_{1}\left(s_{m}\right)\right), \tilde{R}_{1}\right)$ \\
\hline$f_{2}$ & $\left(\tilde{v}_{s_{1}}\left(f_{2}\left(s_{1}\right)\right), \tilde{R}_{1}\right)$ & $\left(\tilde{v}_{s_{2}}\left(f_{2}\left(s_{2}\right)\right), \tilde{R}_{1}\right)$ & -- & $\left(\tilde{v}_{s_{m}}\left(f_{2}\left(s_{m}\right)\right), \tilde{R}_{1}\right)$ \\
\hline$\ldots$ & --- & --- & -- & --- \\
\hline$f_{n}$ & $\left(\tilde{v}_{s_{1}}\left(f_{n}\left(s_{1}\right)\right), \tilde{R}_{1}\right)$ & $\left(\tilde{v}_{s_{2}}\left(f_{n}\left(s_{2}\right)\right), \tilde{R}_{1}\right)$ & & $\left(\tilde{v}_{s_{m}}\left(f_{n}\left(s_{m}\right)\right), \tilde{R}_{1}\right)$ \\
\hline
\end{tabular}

In payoff Table. $1, \tilde{R}_{1}$ is a confidence degree for the value of utility.

As decision maker usually is uncertain about first-order imprecise probabilities, we describe the probabilities of states of nature as Z-numbers (Table.2).

Table.2. Probabilities of states as Z-numbers

\begin{tabular}{|l|l|l|l|}
\hline$\left(\tilde{P}\left(s_{1}\right), \tilde{R}_{2}\right)$ & $\left(\tilde{P}\left(s_{2}\right), \widetilde{R}_{2}\right)$ & $\ldots$ & $\left(\tilde{P}\left(s_{m}\right), \tilde{R}_{2}\right)$ \\
\hline
\end{tabular}

In Table.2, $\tilde{R}_{2}$ is a confidence degree for the value of probability of the state of nature.

Formally the problem is formulated as follows. Decisionmaking under uncertainty can be considered as 4-tuple $\left(S, \tilde{Z}_{X}\right.$, $A, \succeq$ ), where $S=\left\{s_{1}, s_{2}, \ldots, s_{n}\right\}-$ a space of mutually exclusive and exhaustive states of nature, $\tilde{Z}_{X}$ - a set of outcomes, described by Z-valuation. $A$ is the set of actions that are functions $f: S \rightarrow \tilde{Z}_{X}, \succeq$ is the non-additive preference relation on the set of actions. In decision-making under uncertainty, a 
probability over $S$ is imprecise. $F_{S}$ is a $\sigma$-algebra of subsets $B$ of $S$. Denote by $A_{0}$ the set of all $F_{S}$ - measurable step-valued functions from $S$ to $X$ and denote $A_{C}$ the constant actions in $A_{0}$. Let $A$ be a convex subset of $X^{S}$ which includes $A_{C}$. $X$ can be considered as a subset of some linear space, and $X^{s}$ can then be considered as a subspace of the linear space of all functions from $S$ to the first linear space. The problem is to determine preferences among alternatives by means of a utility function.

The suggested decision-making methodology uses Choquet expected utility for description of preferences. The utility function used here is as follows,

$$
\tilde{Z}_{U\left(f_{i}\right)}^{\prime}=\int_{S} \tilde{Z}_{v_{s_{j}}^{\prime}}^{\prime}\left(f_{i}(s)\right) d \tilde{Z}_{\eta}^{\prime}
$$

The decision making problem in this case consists in the determination of an optimal action $f^{*} \in A$ such that

$$
\tilde{Z}_{U\left(f_{i}^{*}\right)}^{\prime}=\max _{f \in A}\left\{\int_{S} \tilde{Z}_{v_{s_{j}}^{\prime}}^{\prime}\left(f_{i}(s)\right) d \tilde{Z}_{\eta}^{\prime}\right\}
$$

\section{A SOLUTION TO THE PROBLEM}

Let outcomes $\tilde{Z}_{v_{s_{j}}}\left(f_{i}\left(s_{j}\right)\right)=\left(\tilde{v}_{s_{j}}\left(f_{i}\left(s_{j}\right)\right), \tilde{R}_{1}\right)$ and the probabilities $\tilde{Z}_{P\left(s_{j}\right)}=\left(\tilde{P}\left(s_{j}\right), \tilde{R}_{2}\right)$ of the states $s_{j} \in S$ where, $\tilde{R}_{1}=\left\{\left(x_{2}, \mu_{\tilde{R}_{1}}(x)\right): x_{2} \in[0,1]\right\}$

and

$\tilde{R}_{2}=\left\{\left(y_{2}, \mu_{\tilde{R}_{2}}(y)\right): y_{2} \in[0,1]\right\}$ are represented by trapezoidal and triangle fuzzy numbers.

In this study it is assumed that it is given only $N L$-described reasonable knowledge about probability distribution over $S$. It means that a state $s_{j}$ is assigned a linguistic probability $\tilde{P}_{j}$ that can be described by Z-number. Initial data for the problem are represented by given linguistic probabilities for $m-1$ states of nature whereas for one of the given states the probability is unknown. So at first it is required to obtain the unknown probability. To determinate an unknown probability of state $s_{j}$ $\tilde{Z}_{P\left(s_{j}\right)}$ on a base of given probabilities $\tilde{Z}_{P\left(s_{1}\right)}, \tilde{Z}_{P\left(s_{2}\right)}, \ldots$, $\tilde{Z}_{P\left(s_{j-1}\right)}, \ldots, \tilde{Z}_{P\left(s_{m}\right)}$ we use the method suggested in [15].

Given the payoff table and the complete probability distribution we can evaluate the values of Choquet integral on base of Eq.(4). For this aim we use computation with Z-numbers which falls within the province of Computing with words. Computation with Z-information in this study is based on converting of Z-numbers $[12,13]$.

To convert the given Z-numbers on outcomes and probabilities first we determine the expected values of fuzzy numbers $R_{1}$ and $R_{2}$ describing reliability of variables of outcome and probability:

$$
\alpha_{1}=\frac{\int x \mu_{\tilde{R}_{1}}(x) d x}{\int \mu_{\tilde{R}_{1}}(x) d x},
$$

$$
\alpha_{2}=\frac{\int y \mu_{\tilde{R}_{2}}(y) d y}{\int \mu_{\tilde{R}_{y}}(y) d y}
$$

Now we can represent the values of variables outcome and probability as: $\tilde{Z}_{v_{s_{j}}}^{\alpha_{1}}\left(f_{i}(s)\right)=\left(a_{1}, a_{2}, a_{3}, a_{4} ; \alpha_{1}\right)$, $\tilde{Z}_{P\left(s_{j}\right)}^{\alpha_{2}}=\left(c_{1}, c_{2}, c_{3} ; \alpha_{2}\right)$. Then we convert this weighted Z-number to fuzzy number: $\tilde{Z}_{v_{s_{j}}}^{\prime}\left(f_{i}(s)\right)=\left(\sqrt{\alpha_{1}} \mathrm{a}_{1}, \sqrt{\alpha_{1}} \mathrm{a}_{2}, \sqrt{\alpha_{1}} \mathrm{a}_{3}, \sqrt{\alpha_{1}} \mathrm{a}_{4} ; 1\right)$, $\tilde{Z}_{P\left(s_{j}\right)}^{\prime}=\left(\sqrt{\alpha_{2}} c_{1}, \sqrt{\alpha_{2}} c_{2}, \sqrt{\alpha_{2}} c_{3} ; 1\right)$.

Given the complete probability distribution we construct lower prevision. The determination of a lower prevision $\tilde{Z}_{v_{s}}^{\prime}(f(s))$ from linguistic probability distribution $\tilde{P}$ has a great role in the determination of the preferences in this model. When the states of nature are just some elements, the measure is defined [15] as,

$$
\tilde{Z}_{\eta_{\tilde{P}}}^{\prime}(H)=\bigcup_{\alpha \in(0,1]} a \cdot\left[\begin{array}{r}
\left.\tilde{Z}_{\eta_{\tilde{P}_{\text {left }}}^{\prime}}{ }^{\alpha}(H), \tilde{Z}_{\eta_{\tilde{P}_{\text {right }}^{\prime}}}{ }^{\alpha}(H)\right], \\
H \subset S=\left\{s_{1}, \ldots, s_{m}\right.
\end{array}\right\},
$$

where, $\quad \tilde{Z}_{\eta_{\tilde{P}}}^{\prime \alpha}(H)=\inf \left\{\sum_{s_{i} \in H} p\left(s_{j}\right), \ldots, p\left(s_{m}\right) \in P^{\alpha}\right\}$,

$$
P^{\alpha}=\left\{\left(p\left(s_{1}\right), \ldots,\left(p\left(s_{m}\right)\right) \in P_{1}^{\alpha} \times \ldots \times P_{m}^{\alpha} \mid \sum_{j=1}^{m} p\left(s_{j}\right)=1\right\},\right.
$$

Here $P_{1}^{\alpha}, \ldots, P_{m}^{\alpha}$ are $\alpha$-cuts of fuzzy probabilities $P_{1}, \ldots, P_{m}$, $p\left(s_{1}\right), \ldots, p\left(s_{m}\right)$ are basic probabilities for $\tilde{P}_{1}, \ldots, \tilde{P}_{m}, \times$ denotes the Cartesian product.

Now we can construct a fuzzy measure with triangle membership function from fuzzy set of possible probability distributions as its lower probability function (lower prevision) taking into consideration Eq.(8) and the method used in [15].

As we have an ordinary fuzzy numbers with trapezoidal and triangular membership functions then we can obtain the fuzzy values of utility function $\tilde{U}(f(s))$ for each alternative by Eq.(2):

$$
\begin{gathered}
\tilde{Z}_{U\left(f_{i}\right)}^{\prime}=\int_{S} \tilde{Z}_{v_{s j}}^{\prime}\left(f_{i}(s)\right) d \tilde{Z}_{\eta}^{\prime} \\
\tilde{Z}_{U\left(f_{i}\right)}^{\prime}=\int_{S}\left(\sqrt{\alpha_{1}} \times \tilde{v}_{s}(f(s) ; 1) d \tilde{Z}_{\eta_{\tilde{P}}^{\prime}}^{\prime}\right. \\
\tilde{Z}_{U\left(f_{i}\right)}^{\prime}=\sum_{j=1}^{m}\left(\tilde{Z}_{\tilde{\eta}_{\tilde{P}}}^{\prime}\left(H_{(j)}\right)-\tilde{Z}_{\eta_{\tilde{P}}}^{\prime}\left(H_{(j-1)}\right)\right) \tilde{Z}_{v_{s_{(j)}}\left(f_{i}(s)\right)} \\
=\sum_{j=1}^{m}\left(\tilde{Z}_{\eta_{\tilde{P}}^{\prime}}\left(H_{(j)}\right)-\tilde{Z}_{\eta_{\tilde{P}}^{\prime}}\left(H_{(j-1)}\right)\right)\left(\sqrt{\alpha_{1}} \times \tilde{v}_{s_{(j)}}\left(f_{i}(s) ; 1\right)\right.
\end{gathered}
$$

An optimal action $f^{*} \in A$ is obtained in accordance with Eq.(5). 


\section{AN APPLICATION TO BUSINESS PROBLEM}

We consider the business problem under imprecise information described by Z-valuation. Suppose a hotel is considering the construction of an additional wing. The possibility of adding $30\left(f_{1}\right), 40\left(f_{2}\right)$ and $50\left(f_{3}\right)$ rooms is evaluating. The success of the addition depends on a combination of local government legislation and competition in the field. There are three states of nature: positive legislation and low competition $\left(s_{1}\right)$, positive legislation and strong competition $\left(s_{2}\right)$, no legislation and low competition $\left(s_{3}\right)$. Also we have the values anticipated payoffs (in percentage). The problem is to find how many rooms to build in order to maximize the return on investment. $Z$-valuation for the utilities of the each act taken at various states and probabilities on states are provided in Table. 3 and Table.4, respectively.

Table.3. The utility values of actions under various states

\begin{tabular}{|c|c|c|c|}
\hline & $\left\{s_{1}\right\}$ & $\left\{s_{2}\right\}$ & $\left\{s_{3}\right\}$ \\
\hline$f_{1}$ & $\{$ high; likely $\}$ & $\begin{array}{c}\{\text { below than high; } \\
\text { likely }\}\end{array}$ & $\{$ medium; likely $\}$ \\
\hline$f_{2}$ & $\begin{array}{c}\{\text { below than high; } \\
\text { likely }\}\end{array}$ & $\{$ low; likely $\}$ & $\begin{array}{c}\{\text { below than high; } \\
\text { likely }\}\end{array}$ \\
\hline$f_{3}$ & $\begin{array}{c}\{\text { below than high; } \\
\text { likely }\}\end{array}$ & $\{$ high; likely $\}$ & $\{$ medium; likely $\}$ \\
\hline
\end{tabular}

Table.4. The values of probabilities of states of nature

\begin{tabular}{|c|c|c|}
\hline $\begin{array}{c}\left\{s_{1}\right\}=\left\{\begin{array}{c}\text { medium; quite } \\
\text { sure }\}\end{array}\right.\end{array}$ & $\begin{array}{l}\left.\qquad s_{2}\right\}=\{\text { more than } \\
\text { medium; quite sure }\}\end{array}$ & $\begin{array}{l}\left\{s_{3}\right\}=\{\text { low; } \\
\text { quite sure }\}\end{array}$ \\
\hline
\end{tabular}

Here $\tilde{Z}_{v_{s_{j}}}\left(f_{i}\left(s_{j}\right)\right)=\left(\tilde{v}_{s_{j}}\left(f_{i}\left(s_{j}\right)\right), \tilde{R}_{1}\right)$, where the outcomes are the trapezoidal fuzzy numbers and corresponding reliability is a triangular fuzzy number:

$$
\begin{aligned}
& \tilde{Z}_{v_{s 1}\left(f_{1}\left(s_{1}\right)\right)}=\left(\tilde{v}_{s_{1}}\left(f_{1}\left(s_{1}\right)\right), \tilde{R}_{1}\right)=\{\text { high; likely }\} \\
& =[(7,8,9,10 ; 1),(0.6,0.7,0.8 ; 1)] \text {, } \\
& \tilde{Z}_{v_{s 2}\left(f_{1}\left(s_{2}\right)\right)}=\left(\tilde{v}_{s_{2}}\left(f_{1}\left(s_{2}\right)\right), \tilde{R}_{1}\right)=\{\text { below than high; likely }\} \\
& =[(6,7,8,9 ; 1),(0.6,0.7,0.8 ; 1)], \\
& \tilde{Z}_{v_{s 3}\left(f_{1}\left(s_{3}\right)\right)}=\left(\tilde{v}_{s_{3}}\left(f_{1}\left(s_{3}\right)\right), \tilde{R}_{1}\right)=\{\text { medium; likely }\} \\
& =[(4,5,6,7 ; 1),(0.6,0.7,0.8 ; 1)] \text {, } \\
& \tilde{Z}_{v_{s 1}\left(f_{2}\left(s_{1}\right)\right)}=\left(\tilde{v}_{s_{1}}\left(f_{2}\left(s_{1}\right)\right), \tilde{R}_{1}\right)=\{\text { below than high; likely }\} \\
& =[(6,7,8,9 ; 1),(0.6,0.7,0.8 ; 1)] \text {, } \\
& \tilde{Z}_{v_{s 2}\left(f_{2}\left(s_{2}\right)\right)}=\left(\tilde{v}_{s_{2}}\left(f_{2}\left(s_{2}\right)\right), \tilde{R}_{1}\right)=\{\text { low; likely }\} \\
& =[(3,4,5,6 ; 1),(0.6,0.7,0.8 ; 1)], \\
& \tilde{Z}_{v_{s 3}\left(f_{2}\left(s_{3}\right)\right)}=\left(\tilde{v}_{s_{3}}\left(f_{2}\left(s_{3}\right)\right), \tilde{R}_{1}\right)=\{\text { below than high; likely }\} \\
& =[(6,7,8,9 ; 1),(0.6,0.7,0.8 ; 1)], \\
& \tilde{Z}_{v_{s 1}\left(f_{3}\left(s_{1}\right)\right)}=\left(\tilde{v}_{s_{1}}\left(f_{3}\left(s_{1}\right)\right), \tilde{R}_{1}\right)=\{\text { below than high; likely }\} \\
& =[(6,7,8,9 ; 1),(0.6,0.7,0.8 ; 1)] \text {, } \\
& \tilde{Z}_{v_{s 2}\left(f_{3}\left(s_{2}\right)\right)}=\left(\tilde{v}_{s_{2}}\left(f_{3}\left(s_{2}\right)\right), \tilde{R}_{1}\right)=\{\text { high; likely }\} \\
& =[(7,8,9,10 ; 1),(0.6,0.7,0.8 ; 1)], \\
& \tilde{Z}_{v_{s 3}\left(f_{3}\left(s_{3}\right)\right)}=\left(\tilde{v}_{s_{3}}\left(f_{3}\left(s_{3}\right)\right), \tilde{R}_{1}\right)=\{\text { medium; likely }\} \\
& =[(4,5,6,7 ; 1),(0.6,0.7,0.8 ; 1)] .
\end{aligned}
$$

Let the probabilities for $s_{1}$ and $s_{2}$ be Z-numbers $\left.\tilde{Z}_{P\left(s_{j}\right)}=\left(\tilde{P}\left(s_{j}\right)\right), \tilde{R}_{2}\right)$, where the probabilities and the corresponding reliability are the triangular fuzzy numbers:

$\left.\tilde{Z}_{P\left(s_{1}\right)}=\left(\tilde{P}\left(s_{1}\right)\right), \tilde{R}_{2}\right)=\{$ medium; quite sure $\}$

$=[(0.25,0.3,0.35 ; 1),(0.8,0.9,1 ; 1)]$.

$\left.\tilde{Z}_{P\left(s_{2}\right)}=\left(\tilde{P}\left(s_{2}\right)\right), \widetilde{R}_{2}\right)=\{$ more than medium; quite sure $\}$

$$
=[(0.35,0.4,0.45 ; 1),(0.8,0.9,1 ; 1)] \text {. }
$$

In accordance with [7] we have calculated probability for $s_{3}$ :

$$
\left.\tilde{Z}_{P\left(s_{3}\right)}=\left(\tilde{P}\left(s_{3}\right)\right), \tilde{R}_{2}\right)=\{\text { low; quite sure }\}
$$$$
=[(0.2,0.3,0.4 ; 1),(0.8,0.9,1 ; 1)] \text {. }
$$

Then we convert the value of fuzzy reliability into a crisp number based on Eq.(5)-Eq.(6):

$$
\begin{gathered}
\alpha_{1}=\frac{\int x \mu_{\tilde{R}_{1}}(x) d x}{\int \mu_{\tilde{R}_{1}}(x) d x}=0.7, \\
\alpha_{2}=\frac{\int y \mu_{\tilde{R}_{2}}(y) d y}{\int \mu_{\tilde{R}_{y}}(y) d y}=0.9
\end{gathered}
$$

Given the complete fuzzy probability distribution $\tilde{P}\left(s_{j}\right), j=\overline{1,3}$, we add the weight of the reliability to the restriction and have the weighted Z-number for the outcomes and the probabilities:

$$
\begin{aligned}
& \tilde{Z}_{v_{s_{1}}\left(f_{1}\left(s_{1}\right)\right)}^{\alpha_{1}}=(7,8,9,10 ; 0.7), \\
& \tilde{Z}_{v_{s_{2}}\left(f_{1}\left(s_{2}\right)\right)}^{\alpha_{1}}=(6,7,8,9 ; 0.7), \\
& \tilde{Z}_{v_{s_{3}}\left(f_{1}(s)\right)}^{\alpha_{1}}=(4,5,6,7 ; 0.7), \\
& \tilde{Z}_{v_{s_{1}}}^{\alpha_{1}}\left(f_{2}\left(s_{1}\right)\right)=(6,7,8,9 ; 0.7), \\
& \tilde{Z}_{v_{s_{2}}}^{\alpha_{1}}\left(f_{2}\left(s_{2}\right)\right)=(3,4,5,6 ; 0.7), \\
& \tilde{Z}_{v_{s_{3}}}^{\alpha_{1}}\left(f_{2}(s)\right)=(6,7,8,9 ; 0.7), \\
& \tilde{Z}_{v_{s_{1}}}^{\alpha_{1}}\left(f_{3}\left(s_{1}\right)\right)=(6,7,8,9 ; 0.7), \\
& \tilde{Z}_{v_{s_{2}}}^{\alpha_{1}}\left(f_{3}\left(s_{2}\right)\right)=(7,8,9,10 ; 0.7), \\
& \tilde{Z}_{v_{s_{3}}}^{\alpha_{1}}\left(f_{3}\left(s_{3}\right)\right)=(4,5,6,7 ; 0.7), \\
& \tilde{Z}_{P\left(s_{1}\right)}^{\alpha_{2}}=(0.25,0.3,0.35 ; 0.9), \\
& \tilde{Z}_{P\left(s_{2}\right)}^{\alpha_{2}}=(0.35,0.4,0.45 ; 0.9), \\
& \tilde{Z}_{P\left(s_{3}\right)}^{\alpha_{2}}=(0.2,0.3,0.4 ; 0.9)
\end{aligned}
$$

Now we convert the obtained weighted numbers to fuzzy numbers: 


$$
\begin{gathered}
\tilde{Z}_{v_{s_{1}}\left(f_{1}\left(s_{1}\right)\right)}^{\prime}=(5.85662,6.69328,7.52994,8.3666 ; 1), \\
\tilde{Z}_{v_{s_{2}}\left(f_{1}\left(s_{2}\right)\right)}^{\prime}=(5.01996,5.85662,6.69328,7.52994 ; 1), \\
\tilde{Z}_{v_{s_{3}}\left(f_{1}\left(s_{3}\right)\right)}^{\prime}=(3.34664,4.1833,5.01996,5.85662 ; 1), \\
\tilde{Z}_{v_{s_{1}}\left(f_{2}\left(s_{1}\right)\right)}^{\prime}=(5.01996,5.85662,6.69328,7.52994 ; 1), \\
\tilde{Z}_{v_{s_{2}}\left(f_{2}\left(s_{2}\right)\right)}^{\prime}=(2.50998,3.34664,4.1833,5.01996 ; 1), \\
\tilde{Z}_{v_{s_{3}}\left(f_{2}\left(s_{3}\right)\right)}^{\prime}=(5.01996,5.85662,6.69328,7.52994 ; 1), \\
\tilde{Z}_{v_{s_{1}}}^{\prime}\left(f_{3}\left(s_{1}\right)\right) \\
\tilde{Z}_{v_{s_{2}}}^{\prime}\left(f_{3}\left(s_{2}\right)\right) \\
\tilde{Z}_{v_{s_{3}}\left(f_{3}\left(s_{3}\right)\right)}^{\prime}=(5.01996,5.85662,6.69328,7.52994 ; 1), \\
\tilde{Z}_{P\left(s_{1}\right)}^{\prime}=(0.237171,0.284605,0.33203915 ; 1), \\
\tilde{Z}_{P\left(s_{2}\right)}^{\prime}=(0.332039,0.379473,0.426907 ; 1), \\
\tilde{Z}_{P\left(s_{3}\right)}^{\prime}=(0.189737,0.284605,0.379473 ; 1)
\end{gathered}
$$

Given these data and following the proposed decision making method, we can obtain an overall utility as a fuzzyvalued Choquet integral:

$$
\begin{aligned}
\tilde{Z}_{U\left(f_{i}\right)}^{\prime}= & \tilde{Z}_{\eta_{\tilde{P}}}^{\prime}\left(\left\{s_{(1)}\right\}-\tilde{Z}_{\eta_{\tilde{P}}}^{\prime}\left\{s_{(0)}\right\}\right) \cdot \tilde{Z}_{v_{s(1)}}^{\prime}\left(f_{i}(s)\right)+ \\
& \tilde{Z}_{\eta_{\tilde{P}}}^{\prime}\left(\left\{s_{(1)}, s_{(2)}\right\}-\tilde{Z}_{\eta_{\tilde{P}}}^{\prime}\left\{s_{(1)}\right\}\right) \cdot \tilde{Z}_{v_{s_{(2)}}}^{\prime}\left(f_{i}(s)\right) \\
& \tilde{Z}_{\eta_{\tilde{P}}}^{\prime}\left(\left\{s_{(1)}, s_{(2)}, s_{(3)}\right\}-\tilde{Z}_{\eta_{\tilde{P}}}^{\prime}\left\{s_{(1)}, s_{(2)}\right\}\right) \cdot \tilde{Z}_{v_{s_{(3)}}}^{\prime}\left(f_{i}(s)\right)
\end{aligned}
$$

The states are ordered such that:

For the $1^{\text {st }}$ alternative, $\tilde{Z}_{v_{s_{1}}\left(f_{1}\left(s_{1}\right)\right)}^{\prime} \succ \tilde{Z}_{v_{s_{2}}\left(f_{1}\left(s_{2}\right)\right)}^{\prime} \succ \tilde{Z}_{v_{s_{3}}\left(f_{1}\left(s_{3}\right)\right)}^{\prime}$,

For the $2^{\text {nd }}$ alternative, $\tilde{Z}_{v_{s_{1}}\left(f_{1}\left(s_{1}\right)\right)}^{\prime} \succ \tilde{Z}_{v_{s_{3}}\left(f_{1}\left(s_{3}\right)\right)}^{\prime} \succ \tilde{Z}_{v_{s_{2}}\left(f_{1}\left(s_{2}\right)\right)}^{\prime}$,

For the $3^{\text {rd }}$ alternative, $\tilde{Z}_{v_{s_{2}}\left(f_{1}\left(s_{2}\right)\right)}^{\prime} \succ \tilde{Z}_{v_{s_{1}}\left(f_{1}\left(s_{1}\right)\right)}^{\prime} \succ \tilde{Z}_{v_{s_{3}}\left(f_{1}\left(s_{3}\right)\right)}^{\prime}$.

The $\alpha$-cuts of $\tilde{Z}_{\tilde{\eta}_{\tilde{P}}}^{\prime}\left(\left\{s_{1}, s_{2}\right\}, \tilde{Z}_{\tilde{\eta}_{\tilde{p}}}^{\prime}\left(\left\{s_{1}, s_{3}\right\}\right.\right.$ are found as the solutions of Eq.(8).

So we can determine the triangular fuzzy numbers

$$
\begin{aligned}
& Z_{\eta_{\tilde{P}}^{\prime}}^{\prime}\left(\left\{s_{1}, s_{2}\right\}\right)=(0.620528,0.715396,0.715396) \\
& Z_{\eta_{\tilde{P}}^{\prime}}\left(\left\{s_{1}, s_{3}\right\}\right)=(0.573094,0.620528,0.620528)
\end{aligned}
$$

Given this, the values of the utility function for the alternatives are as follows:

$$
\begin{aligned}
& \tilde{Z}_{U\left(f_{1}\right)}^{\prime}=(-0.75021,5.618504,6.454851,11.7923) \\
& \tilde{Z}_{U\left(f_{2}\right)}^{\prime}=(-1.99406,5.532631,6.369291,13.30069)
\end{aligned}
$$

$$
\tilde{Z}_{U\left(f_{3}\right)}^{\prime}=(-0.11198,6.296418,7.212137,12.66864)
$$

Ranking of fuzzy values of utilities gives a preference to the third alternative, i.e. $f_{3} \succ f_{1} \succ f_{2}$.

\section{CONCLUSION}

An important qualitative attribute of information on which decisions are based is its reliability. Unfortunately in almost all existing decision theories reliability of decision relevant information is missing. In this study, we have considered a problem of decision making under Z-valued information represented by Z-number which induces a possibility distribution over probability distributions associated with decision variables. We developed method of decision making which associates with the construction of a non-additive measure as a lower prevision and uses this capacity in Choquet integral for constructing a utility function in $\mathrm{Z}$-valuation environment. Computation with $\mathrm{Z}$-information is based on conversion of Z-numbers. The outlined approach to decision-making brings forward a much more general framework that coincides with human-oriented assessment of imperfect information. We applied the suggested theory and methodology to solving a real world business problem, which proved its validity.

\section{REFERENCES}

[1] John von Neumann and O. Morgenstern, "Theory of Games and Economic Behaviour", Second Edition, Princeton University Press, 1947.

[2] L. J. Savage, "The Foundations of Statistics", JohnWiley \& Sons, 1954.

[3] Paul Anand, Prasanta Pattanaik and Clemens Puppe, "The Handbook of Rational and Social Choice", Oxford University Press, 2009.

[4] J. Montero and D. Ruan, "Modelling uncertainty", Information Sciences, Vol. 180, No. 6, pp. 799-802, 2010.

[5] S. Sumathi and Surekha Paneerselvam, "Computational Intelligence Paradigms Theory and Applications using MATLAB", CRC Press, 2010.

[6] P. Walley, "Statistical inferences based on a second-order possibility distribution", International Journal of General Systems, Vol. 26, No. 4, pp. 337-383, 1997.

[7] R. A. Aliev, W. Pedrycz, O. H. Huseynov and L. M. Zeinalova, "Decision making with second order information granules", Granular Computing and Intelligent Systems, Vol. 13, pp. 117-153, 2011.

[8] Gert De Cooman, "Precision-Imprecision Equivalence in a Broad Class of Imprecise Hierarchical Uncertainty Models", Journal of Statistical Planning and Inference, Vol.105, No. 1, pp. 175-198, 2002.

[9] P. Meyer and M. Roubens, "On the use of the Choquet integral with fuzzy numbers in multiple criteria decision support", Fuzzy Sets and Systems, Vol. 157, No. 7, pp. 927-938, 2006.

[10] Lotfi A. Zadeh, "A note on a Z-number", Information Sciences, Vol. 181, pp. 2923-2932, 2011. 
[11] Ronald R. Yager, "On Z-valuations using Zadeh`s Znumbers", International Journal of Intelligent Systems, Vol. 27, No. 3, pp. 259-278, 2012.

[12] Bingyi Kang, Daijun Wei, Ya Li and Yong Deng, "A method of converting Z-number to classical fuzzy number", Journal of Information and Computational Science, Vol. 9, No. 3, pp. 703-709, 2012.

[13] Bingyi Kang, Daijun Wei, Ya Li and Yong Deng, "Decision Making Using Z-numbers under Uncertain Environment", Journal of Computational Information Systems, Vol. 8, No. 7, pp. 2807-2814, 2012.

[14] R. A. Aliev, B. Fazlollahi and R. R. Aliev, "Soft Computing and Its Applications in Business and Economics", Springer, 2004.

[15] R. Aliev, W. Pedrycz, B. Fazlollahi, O. H. Huseynov, A. V. Alizadeh and B. G. Guirimov, "Fuzzy logic-based generalized decision theory with imperfect information", Information Sciences, Vol. 189, pp. 18-42, 2012.

[16] F. Modave and D. Iourinski, "Axiomatization of Qualitative Multicriteria Decision Making with the Sugeno Integral", Intelligent Systems Design and Applications, Advances in Soft Computing. Vol. 23, pp. 77-86, 2003.

[17] R. Yang, Z. Wang, P. A. Heng and K. S. Leung, "Fuzzified Choquet integral with a fuzzy valued integrand and its application on temperature prediction", IEEE Transactions on Systems, Man and Cybernetics, Part B, Vol. 38, No. 2, pp. 367-380, 2008.

[18] E. Schmitt, V. Bombardier and L. Wendling, "Improving fuzzy rule classifier by extracting suitable features from capacities with respect to the Choquet integral", IEEE Transactions on Systems, Man and Cybernetics, Vol. 38, No. 5, pp. 1195-1206, 2008.
[19] Zengwu Wang and Jia-an Yan, "Choquet integral and its applications: a survey", Academy of Mathematics and Systems Science, 2006.

[20] R. Alo, A. de Korvin and F. Modave, "Using fuzzy functions to select an optimal action in decision theory", North American Fuzzy Information Processing SocietyFLINT Conference, pp. 348 -353, 2002.

[21] R. R. Yager, "Uncertainty modeling and decision support", Reliability Engineering and System Safety, Vol. 85, No. 13, pp. 341-354, 2004.

[22] I. Levi, "On indeterminate probabilities", Journal of Philosophy, Vol. 71, No. 13, pp. 391-418, 1974.

[23] R. A. Aliev and R. R. Aliev, "Soft Computing and its Applications", World Scientific Publishing, 2001.

[24] Gert de Cooman, Matthias C. M. Troffaes and E. Miranda, "n-monotone lower previsions and lower integrals", Proceedings of Fourth International Symposium on Imprecise Probabilities and their Applications, Vol. 16, No. 4, pp. 253-263, 2005.

[25] R. A. Aliev, S. M. Jafarov, L. A. Gardashova and L. M. Zeinalova, "Principles of Decision Making And Control Under Uncertainty", Nargiz Baku, 1999.

[26] H. T. Nguyen and E. A. Walker, "A First Course in Fuzzy Logic", CRC Press, 2000.

[27] E. Miranda and M. Zaffalon, "Conditional models: coherence and inference through sequences of joint mass functions", Journal of Statistical Planning and Inference, Vol. 140, No. 7, pp. 1805-1833, 2010.

[28] P. Walley, "Measures of uncertainty in expert systems", Artificial Intelligence, Vol. 83, No. 1, pp.1-58, 1996. 